Research Article

\title{
Application of Multivariate Optimization for Phenolic Compounds and Antioxidants Extraction from Moroccan Cannabis sativa Waste
}

\author{
Smail Aazza $\mathbb{D}$ \\ Laboratory of Phytochemistry, National Agency of Medicinal and Aromatic Plants, Morocco \\ Correspondence should be addressed to Smail Aazza; aazzasmail@ymail.com
}

Received 5 June 2021; Revised 26 July 2021; Accepted 12 August 2021; Published 21 August 2021

Academic Editor: Ramon Gerardo Guevara-González

Copyright ( 2021 Smail Aazza. This is an open access article distributed under the Creative Commons Attribution License, which permits unrestricted use, distribution, and reproduction in any medium, provided the original work is properly cited.

\begin{abstract}
A statistical simplex centroid design methodology was applied to determine the effects of different solvents and their mixtures on the yield, total polyphenol content, 2'2-dipheny-l-picrylhydrazyl (DPPH) radical scavenging activity, and ferric reducing antioxidant power (FRAP) of extracts from the waste of Cannabis sativa. The different extractor solvents (ethanol, methanol, water, and hexane) and their binary and ternary combinations were evaluated. The experimental results and their response surface models showed that the highest TPC yield values occur with the binary interaction between water and ethanol around the proportion of (ethanol, 70\%; water, 30\%). The desirability function showed that the optimal conditions were for TPC extraction ternary mixtures which consisted of $75 \%$ ethanol, $12.5 \%$ methanol, and $12.5 \%$ water. Ternary mixtures including water and binary mixture (ethanol $50 \%$ to $75 \%$ ) yielded extracts with the best DPPH antioxidant activity, whereas pure methanol was the best solvent for extracting molecules with FRAP antioxidant capacity. The desirability function including all responses showed that the optimal solvent mixture consisted of $25 \%$ ethanol and $75 \%$ methanol.
\end{abstract}

\section{Introduction}

Cannabis sativa $\mathrm{L}$. is a dioecious plant belonging to the Cannabaceae family. Cannabinoids, flavones, and terpenes are the main phytochemicals found in this plant [1]. This plant was used as a medicine before the Christian era in Asia, principally in India, and after it was introduced to Western medicine in the midst of the 19th century. Recently, the interest in C. sativa $\mathrm{L}$. has drastically increased, due to psychoactive and nonpsychoactive compounds, namely, $\Delta$ 9-tetrahydrocannabinol $(\triangle 9$-THC) and cannabidiol (CBD) [2]. This interest had increased in the 1990s through the description of cannabinoid receptors and the identification of an endogenous cannabinoid system in the brain [3]. In the last years, Cannabis sativa L. has been the center of the attention of the scientific community and definitely became one of the most studied plants. In Morocco, cannabis cultivation in the northern Rif region is currently undergoing its most significant evolution since the hashish industry emerged in the 1960s and dramatically developed in the 1980s [4]. To separate the golden beige powder called "Hashish" resulting from the solidification of the droplets of resin exuding from the tops of the female plants, these plants are first dried, and the resin and trichomes become dusty and more brittle; thus the resin can be separated from the plant material by beat or shake over a thin stretched nylon veil which acts as a sieve $[5,6]$. After this process, the plant residue (whole plant fragments) is separated from seeds and discarded.

Phenolic compounds are associated with a high number of biological activities including antioxidant capacity, which may help to protect the cells against the oxidative damage caused by free radicals [7]. Several novel extraction techniques have been developed in an attempt to obtain a more efficient extraction of target compounds by reducing both extraction time and used solvent [8]. The efficient recovery of bioactive polyphenolic phytochemicals, which occur widely in several agri-food residues that may serve as both 
food additives and bioactive substances in cosmetics and pharmaceuticals, is one of the higher value options [9]. These secondary metabolites, which usually occur in low concentrations, are medicinally useful. The yields of these metabolites depend on the solvents and methods of extraction [10]. The quantity and quality of plant extracts are depending on the extraction protocol, which includes several factors such as the type of solvent, temperature, $\mathrm{pH}$, the number of extraction steps, liquid-to-solid ratio, and the particle size of the solute contributing to the efficacy of the extraction process [11]. Solvent extraction is the most frequently used technique for the isolation of plant antioxidant compounds, due to the presence of different antioxidant compounds of varied chemical characteristics and polarities. Polar solvents like methanol and ethanol have been extensively employed to extract antioxidant compounds from various plants and plant-based foods [12].

Response surface methodology (RSM) is a wellestablished tool for the optimization of analytical methods, which is widely applied for the analysis of foods and herbal medicine [13]. The mixture design is a class of response surface experiments whose aim is to develop better or innovative formulations providing optimal requests and to create general conceptions about responses and interactions between independent factors allowing the modelization of the studied interaction [9]. To our knowledge, its use to study the effect of solvent combinations on cannabis waste has not been previously reported.

The goal of this experiment is to perform the lowest number of experiments while gaining the maximum amount of data for the development of an efficient and reproducible model with the desired properties, to obtain clear information on the proportions of individual solvents including ethanol, methanol, water, and hexane in a mixture characterized by its capacity to yield extracts with the highest antioxidant activity and containing the greatest amounts of TPC from Cannabis sativa plant residue.

\section{Materials and Methods}

2.1. Extraction. Cannabis sativa plant residue without resin was collected from farmers and was ground into fine powder. The extracts were prepared in triplicate by adding $1 \mathrm{~mL}$ of the solvent (pure solvent and mixture) to $50 \mathrm{mg}$ of plant residue powder and subjected to sonication for 30 minutes in an ultrasonic bath at ambient temperature, so the extracts were recovered after centrifugation for 10 minutes at $10,000 \mathrm{rpm}$ and stored in the dark at $4^{\circ} \mathrm{C}$.

2.2. Total Phenolic Compounds (TPC). Total phenolic content (TPC) was determined by spectrophotometry, using the colorimetric method, based on the Folin-Ciocalteu reagent [14] with modifications. $50 \mu \mathrm{L}$ of extract or gallic acid standard solution was mixed with $450 \mu \mathrm{L}$ of Folin-Ciocalteu reagent solution diluted 10 times. Subsequently, the mixture was shaken on a vortex mixer and left to incubate for 5 minutes at room temperature, and then $450 \mu \mathrm{L}$ of a $\mathrm{Na}_{2} \mathrm{CO}_{3}$ solution $\left(75 \mathrm{~g} \cdot \mathrm{L}^{-1}\right)$ was added and the mixture was shaken again. After incubation for 2 hours at room temperature their absorption was measured at $760 \mathrm{~nm}$ in a UV/visible Jenway 6505 scanning spectrophotometer. The gallic acid calibration curve (standard curve equation, $\left.y=2.8388 x+0.0556, \quad R^{2}=0.9994\right)$ was prepared with a concentration ranging from 0.062 to $1 \mathrm{mg} \cdot \mathrm{mL}^{-1}$ in ethanol. The results are expressed in gallic acid equivalent (GAE) $\mathrm{g}^{-1}$ dry plant mg. All analyses were done in triplicate.

2.3. DPPH Free Radical Scavenging Activity. The free radical scavenging activity of all extracts was performed following the procedure described by Brand-Williams et al. [15]. Briefly, $25 \mu \mathrm{L}$ of each extract (with different dilutions) was added to $1 \mathrm{~mL}$ of an ethanol solution of DPPH $(60 \mu \mathrm{M})$. The absorption measurements were made at $515 \mathrm{~nm}$, after the incubation time of 60 minutes at room temperature. The absorption of a blank sample containing the same amount of ethanol and DPPH solution served as a negative control. The percentage inhibition of activity trapping free radicals in each extract was calculated as follows:

$$
\%_{\text {Inhibition }}=\left[\left(\frac{\mathrm{Abs}_{\text {control }}-\mathrm{Abs}_{\text {sample }}}{\mathrm{Abs}_{\text {control }}}\right) \times 100\right] \text {. }
$$

Triple measurements were made for each sample and the results were expressed in terms of average \pm standard error.

2.4. Ferric Reducing Antioxidant Power (FRAP). The reductive capacity of liquid extracts was determined by the reducing power test described by Oyaizu [16]. This method is based on the transformation of ions, $\mathrm{Fe}^{2+}$ into $\mathrm{Fe}^{3+}$ by polyphenol antioxidants. $0.2 \mathrm{~mL}$ of the extract was mixed with $1 \mathrm{~mL}$ of $0.2 \mathrm{M}$ phosphate buffer and $1 \mathrm{~mL} 1 \%$ potassium ferricyanide in glass tubes. The prepared reaction mixture was incubated for 20 minutes at $50^{\circ} \mathrm{C}$ in a water bath. After incubation, $1 \mathrm{~mL}$ of $10 \%$ trichloroacetic acid solution was added to the reaction mixture. Tubes were centrifuged at $3000 \mathrm{rpm}$ for 10 minutes after $2 \mathrm{~mL}$ of the supernatant was mixed with $2 \mathrm{~mL}$ of bidistilled water and $0.4 \mathrm{~mL}$ of $0.1 \%$ ferric chloride solution. The absorbance was measured at a wavelength of $700 \mathrm{~nm}$. The reductive capacity of the extracts is expressed as ascorbic acid equivalent (Acs. E) $\mathrm{g}^{-1}$ dry plant. All analyses were done in triplicate.

2.5. Experimental Design and Optimization. Mixture models were revealed to be useful tools in describing the behavior of solvent mixtures to extract bioactive compounds from plants [17]. In the present study, a simplex centroid design with a response surface methodology was employed to determine the effect of interactions among extraction solvents on TPC and antioxidants activities to optimize the extraction conditions. Mixture design experiments were designed and analyzed using the free version StatSoft, Inc. (2011), STATISTICA (data analysis software system), version 10. A total of seven combinations are used. Results were expressed as mean \pm SD (RSD (\%)) and were analyzed using ANOVA. Significant differences were determined by Tukey's test, with $p<0.05$ as the significance criterion. The following 
polynomial equation of function Xi was fitted for each factor assessed at each experimental point:

$$
\begin{aligned}
Y= & \beta_{1} X_{1}+\beta_{2} X_{2}+\beta_{3} X_{3}+\beta_{12} X_{1} X_{2} \\
& +\beta_{13} X_{1} X_{3}+\beta_{23} X_{2} X_{3}+\beta_{123} X_{1} X_{2} X_{3}
\end{aligned}
$$

where $Y$ is the predicted response and $\beta 1, \beta 2, \beta 3, \beta 12, \beta 13$, and $\beta 23$ are constant coefficients for each linear and nonlinear interaction term.

Principal component analysis (PCA) was carried out to understand the influence of the extraction solvents on the antioxidant compounds and identify the relationships between the variables.

\section{Results and Discussion}

3.1. Solvent Screening. Solvent selection is one of the most important steps in extraction of phenolics and other bioactive compounds from fruits, vegetables, byproducts, and their recovery from plant-based materials [3]. Generally, desired compound extraction efficiency is influenced by multiple parameters, such as temperature, time, and solvent polarity, and their effects may either be independent or interactive [18]. The results of total phenolic contents and the antioxidant activity of the seven pure solvent extracts are shown in (Figure 1). It is obvious to note that the phenolic compounds and antioxidant capacity of $C$. sativa extracts were highly and significantly influenced $(p<0.05)$ by the type of extraction solvent. The same conclusions have been reported by Liyanapathirana and Shahidi [19]. Estimated values of total phenolic content in different extracts yielded using different solvents ranged from $1.90 \pm 00$ to $19.07 \pm 00 \mathrm{mg} \mathrm{GAE} / \mathrm{g} \mathrm{DP}$, pointing out that ethanol extract was done ten times more TPC than ethyl acetate. Other works corroborating our findings reported TPC results for C. sativa ranging from 5.85 to $17.05 \mathrm{mg} \mathrm{GAE} / \mathrm{g} \mathrm{dw}$ [20]. However, higher TPC in inflorescence samples from industrial hemp was quantified in the range of 10.51 to $52.58 \mathrm{mg} \mathrm{GAE} / \mathrm{g}$ [21].

Ethanol extract exhibited the highest phenolic content followed by methanol extract. Wile, water, and hexane come, respectively, in third and fourth place, whereas dichloromethane was the less efficient solvent for extraction of TPC from Cannabis sativa waste. According to [22], the extract obtained by $100 \%$ ethanol from L. aromatica, showed the highest phenolic content and exhibited the highest total antioxidant activity, reducing power, and $\mathrm{DPPH}$ radical scavenging activity.

The seven solvents were also screened using two antioxidant methods. According to the total antioxidant activity (Figure 1), methanol extracts demonstrated the best antioxidant activity (73.47 mg Asc.E/g DP), followed by ethanol. Conversely, dichloromethane extracts exhibited the lowest antioxidant activity. As shown in Figure 1, the DPPH free radical scavenging activity revealed that ethanol and methanol were the best solvents for extracting molecules endowed with antioxidant properties followed by hexane and then water. According to this screening test, methanol, ethanol, water, and hexane were the best solvents for extracting phenolic compounds and other molecules with antioxidant activity, which allowed us to select them to perform the solvent mixture design.

3.2. Mixture Design. RSM is considered the best statistical approach to assess the influence of different experimental factors and their linear and quadratic interactions on analytical responses and to model and optimize the extraction conditions of phenolics that display important biological activities [3]. This approach gathers the maximum amount of information in the minimum number of analyses. The first mixture design was perfumed using hydroalcoholic solvents by mixing water, ethanol, and methanol, whereas the second was done using three organic solvents, ethanol, methanol, and hexane. The results of the effects of the solvent composition on the yield of TPC and antioxidant activities are shown in Table 1.

3.3. Analysis of Variance (ANOVA). To improve the extraction conditions allowing the best antioxidant compounds recovery, the simplex centroid mixture design was used to construct a contour plot by regression model analysis. Experimental data were fitted to the linear, quadratic, and special cubic models. The statistical significance of the model and equation terms was analyzed based on $p$ value (Prob $>F)$. The goodness of fit of the model to justify its robustness was evaluated by the coefficient of determination $\left(R^{2}\right)$ and the adjusted correlation coefficient $\left(\operatorname{Adj}-R^{2}\right)$, whereas the model's significance was checked using the $F$ test.

The analysis of variance (ANOVA) results based at $95 \%$ confidence interval, including regression model terms, $R^{2}, F$ test, and probability values are depicted in Table 2 . In all mixtures, the linear model explained the variance up to good levels, and expanding from linear to a quadratic and special cubic model improved the fit for the regression analysis. Concerning the hydroalcoholic mixtures, the coefficient of determination $\left(R^{2}\right)$ value of the quadratic model was $\left(R^{2}=1\right)$ and the adjusted $R^{2}$ was 0.92 for both TPC and DPPH assays. In the case of FRAP assay, $R^{2}$ and adjusted $R^{2}$ were equal to 0.96 and 0.95 , respectively, which indicated that the model adequately represented the real relationship between the parameters chosen. Furthermore, those two coefficients were equal to $1\left(R^{2}=1\right)$ for the three responses in the special cubic model. Hence, this special cubic model was chosen since it could explain $100 \%$ of the variability for TPC, DPPH, and FRAP assays, as well as presenting small $p$ values ( $p$ value $<0.000001$ ) and high $F$ values (Table 3 ) indicating that this model was very good in predicting the behavior of the mixtures.

Regarding the mixtures where only organic solvents (ethanol, methanol, and hexane) were used, ANOVA indicated that the quadratic model explained $91 \%, 100 \%$, and $99 \%$ of the total variation of the results, for TPC, DPPH, and FRAP, respectively, but this model was not significant for total phenolic contents ( $p$ value $=0.08$ ), whereas the special cubic model explained the result's variability at $100 \%$ level for all responses, besides 


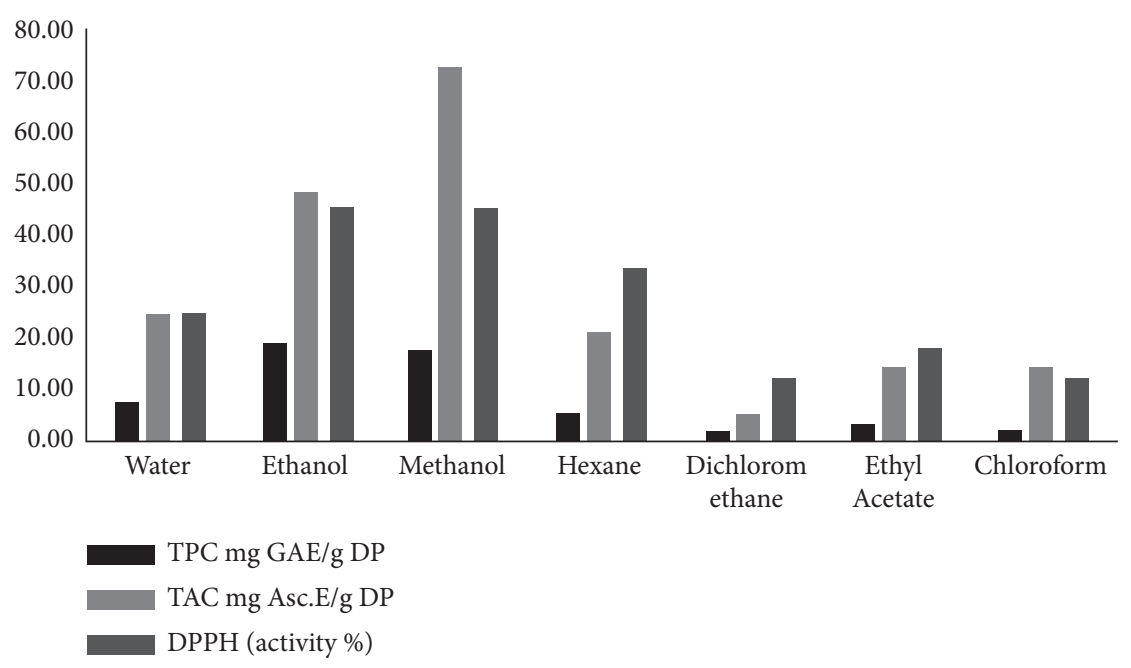

Figure 1: TPC, DPPH, and FRAP of the screened pure solvents.

TABLE 1: Simplex centroid design and response mean values.

\begin{tabular}{lccccccc}
\hline & Ethanol & Methanol & Water & Hexane & TPC & DPPH & FRAP \\
\hline 1 & 0.00 & 0.00 & 0.00 & 100.00 & $5.17 \pm 0.03$ & $34.06 \pm 0.07$ & $21.66 \pm 0.36$ \\
2 & 0.00 & 0.00 & 100.00 & 0.00 & $7.23 \pm 0.25$ & $25.03 \pm 0.21$ & $25.00 \pm 0.09$ \\
3 & 0.00 & 50.00 & 0.00 & 50.00 & $11.66 \pm 0.03$ & $41.03 \pm 0.11$ \\
4 & 0.00 & 50.00 & 50.00 & 0.00 & $15.59 \pm 0.71$ & $46.87 \pm 0.21$ & $35.82 \pm 2.44$ \\
5 & 0.00 & 100.00 & 0.00 & 0.00 & $17.42 \pm 0.14$ & $45.48 \pm 0.18$ & $73.47 \pm 0.38$ \\
6 & 33.33 & 33.33 & 0.00 & 33.33 & $11.97 \pm 0.13$ & $42.45 \pm 0.37$ \\
7 & 33.33 & 33.33 & 33.33 & 0.00 & $20.89 \pm 0.09 \mathrm{ab}$ & $53.93 \pm 0.15$ \\
8 & 50.00 & 0.00 & 0.00 & 50.00 & $16.79 \pm 0.15$ & $36.90 \pm 0.11$ & $50.82 \pm 0.59$ \\
9 & 50.00 & 0.00 & 50.00 & 0.00 & $21.03 \pm 0.08 \mathrm{a}$ & $51.90 \pm 0.10$ \\
10 & 50.00 & 50.00 & 0.00 & 0.00 & $20.14 \pm 0.30$ & $47.21 \pm 0.10$ & $45.08 \pm 0.10$ \\
11 & 100.00 & 0.00 & 0.00 & 0.00 & $19.07 \pm 0.04$ & $45.78 \pm 0.28$ \\
\hline
\end{tabular}

TABLE 2: ANOVA analysis of the three responses.

\begin{tabular}{|c|c|c|c|c|c|c|c|c|}
\hline & \multicolumn{4}{|c|}{ Hydroalcoholic mixtures } & \multicolumn{4}{|c|}{ Organic mixtures } \\
\hline & $F$ & $p$ & $R$-Sqr & Adjusted & $F$ & $p$ & $R$-Sqr & Adjusted \\
\hline \multicolumn{9}{|l|}{$T P C$} \\
\hline Linear & 12.70 & 0.000363 & 0.59 & 0.54 & 54.55 & 0.000000 & 0.86 & 0.84 \\
\hline Quadratic & 448.03 & 0.000000 & 1.00 & 0.99 & 2.71 & 0.082195 & 0.91 & 0.88 \\
\hline Special cubic & 4.85 & 0.044967 & 1.00 & 1.00 & 1765.19 & 0.000000 & 1.00 & 1.00 \\
\hline \multicolumn{9}{|l|}{$\mathrm{DPPH}$} \\
\hline Linear & 5.63 & 0.012586 & 0.38 & 0.32 & 106.30 & 0.000000 & 0.92 & 0.91 \\
\hline Quadratic & 633.22 & 0.000000 & 1.00 & 0.99 & 94.30 & 0.000000 & 1.00 & 0.99 \\
\hline Special cubic & 187.75 & 0.000000 & 1.00 & 1.00 & 23.56 & 0.000256 & 1.00 & 1.00 \\
\hline \multicolumn{9}{|l|}{ FRAP } \\
\hline Linear & 47.72 & 0.000000 & 0.84 & 0.82 & 97.26 & 0.000000 & 0.92 & 0.91 \\
\hline Quadratic & 17.39 & 0.000038 & 0.96 & 0.95 & 25.52 & 0.000004 & 0.99 & 0.98 \\
\hline Special cubic & 480.67 & 0.000000 & 1.00 & 1.00 & 63.60 & 0.000001 & 1.00 & 1.00 \\
\hline
\end{tabular}

presenting small $p$ values $(p$ value $<0.000001)$ and high $F$ values (Table 3 ), indicating that this proposed model provided a very good fit to the data.
The polynomial models describing the correlation between responses obtained for the quadratic model in hydroalcoholic mixtures are as follows: 
TABLE 3: Regression coefficients of second-order polynomial model for the three responses.

\begin{tabular}{|c|c|c|c|c|c|}
\hline & SS & $\mathrm{d} f$ & MS & $F$ & $p$ \\
\hline \multicolumn{6}{|c|}{ Model: hydroalcoholic mixtures } \\
\hline TPC & 426.66 & 6 & 71.11 & 683.86 & 0.000000 \\
\hline DPPH & 1605.17 & 6 & 267.53 & 6976.60 & 0.000000 \\
\hline FRAP & 4067.47 & 6 & 677.91 & 2324.71 & 0.000000 \\
\hline \multicolumn{6}{|c|}{ Model: organic mixtures } \\
\hline TPC & 503.617 & 6 & 83.94 & 3226.01 & 0.000000 \\
\hline DPPH & 430.456 & 6 & 71.74 & 1590.37 & 0.000000 \\
\hline FRAP & 5330.570 & 6 & 888.43 & 929.76 & 0.000000 \\
\hline
\end{tabular}

TPC $=19.04 *$ ethanol $+17.38 *$ methanol $+7.20 *$ water $+8.28 *$ ethanol $*$ methanol $+32.21 *$ ethanol * water $+13.75 *$ methanol * water $+14.18 *$ ethanol * methanol * water +0

DPPH $=45.64 *$ ethanol $+45.34 *$ methanol $+24.89 *$ water $+9.01{ }^{*}$ ethanol ${ }^{*}$ methanol $+68.68 *$ ethanol * water $+49.14 *$ methanol * water $+53.39 *$ ethanol * methanol * water +0

$\mathbf{F R A P}=48.30 *$ ethanol $+72.88 *$ methanol $+24.40 *$ water $-15.94 *$ ethanol * methanol $+44.43 *$ ethanol * water $-33.44 *$ methanol * water $+235.60 *$ ethanol * methanol $*$ water +0

In general, a positive sign for the coefficient in the fitted model indicates the ability of the variable to increase the response, whereas the negative sign indicates the ability of a variable to decrease the response [17]. The equation model showed that the phenolic content and antioxidants activities (DPPH and FRAP) were positively and linearly influenced by ethanol, methanol, and water, respectively, noting that, in linear trials, water resulted in the lowest coefficient, thus, the smallest TPC amounts and the lowest antioxidant activities. Among the binary interactions, water and ethanol interactions resulted in the highest positive effect in all responses, followed by water-methanol interactions for TPC and $\mathrm{DPPH}$, whereas, in the case of FRAP assay, this coefficient was negative in the binary interactions of methanol-ethanol and water-methanol indicating an antagonistic effect between the two solvents. In addition, the ternary mixtures influenced positively the extraction of TPC and antioxidant compounds; this effect was much higher in the FRAP assay.

The regression equations obtained for the special cubic model in organic mixtures correlating the three variables and the analytical responses are as follows:

TPC $=19.07 *$ ethanol $+17.42 *$ methanol $+5.17 *$ hexane $+7.57 *$ ethanol ${ }^{*}$ methanol $+18.66^{*}$ ethanol * hexane $+1.47^{*}$ methanol * hexane $-134.86^{*}$ ethanol * methanol * hexane

DPPH $=45.78 *$ ethanol $+45.48 *$ methanol $+34.06 *$ hexane $+6.31 *$ ethanol $*$ methanol $-12.09 *$ ethanol * hexane $+5.06 *$ methanol $*$ hexane $+20.51 *$ ethanol * methanol * hexane

FRAP $=48.89^{*}$ ethanol $+73.47^{*}$ methanol $+21.66 *$ hexane $-27.83^{*}$ ethanol ${ }^{*}$ methanol $-18.13 *$ ethanol * hexane $+32.99^{*}$ methanol ${ }^{*}$ hexane $-155.13 *$ ethanol

* methanol * hexane

Regarding the linear interaction, in contrast to hexane, ethanol exhibited the highest positive effect on TPC extraction and DPPH free radical scavenging activity, while methanol demonstrated the highest positive influence in the FRAP assay. The strongest positive effect of binary interaction was found in ethanol-hexane mixtures for TPC and $\mathrm{DPPH}$ and methanol-hexane mixtures for FRAP assay, whereas the ternary mixtures had high and negative influence and displayed an antagonistic effect among the components of the mixture for TPC extraction and FRAP assay of the yielded extracts. These results clearly indicate that the extraction is affected by the polarity of the solvents.

\subsection{Contour Plots Analysis}

3.4.1. Total Phenolic Compounds. Among the major classes of plant chemicals, namely, terpenoids, phenolics, and alkaloids phenolic compounds including phenolic acids (hydroxybenzoic and hydroxycinnamic acids), polyphenols (hydrolyzable and condensed tannins), and flavonoids are the most important for dietary applications and the most extensively researched [22]. C. sativa inflorescence could be considered as a potential novel source of polyphenols intended for nutraceutical formulations [21]. The recovery of phenols depends on the solvent system used since the solvent mixture extracts higher TPC amounts than did pure solvent [23]. Thus, the development of a selective method for the extraction of phenolic compounds is required. The threedimensional (3D) interaction contour plots are given in Figure 2(a) as a function of the ethanol, methanol, and water interactions. These three-dimensional response surface plots can exhibit how the initial factors affected different responses more clearly.

The interpretation of contour plots in Figure 2(a) demonstrated the total polyphenols extraction using the combination of ethanol and water resulted in the highest antioxidant activities, which can confirm a synergistic effect between these two solvents, in which the interaction between them demonstrates a better result than their isolated actions. Adding water to organic solvents improves extraction rate, but too high water content brought an increased concomitant extraction of other compounds and then lower phenols concentrations [23]. We also noticed that joining water to methanol enhanced its ability to extract phenolic compounds. The highest TPC yield value on the contour graph is seen to occur with the binary interaction between water and ethanol around the proportion of ethanol $70 \%$, water $30 \%$, whereas the best solvent stated by other authors, for extraction of phenolic compounds from hemp, was $50 \%$ ethanol [20]. According to Wong et al. [24], increasing ethanol concentration from $20 \%$ to $80 \%$ was favorable to increase total phenolic content recovery from palm kernel byproducts from 7.8 to $11.5 \mathrm{mg} / \mathrm{g}$ GAE.

According to the desirability analysis (results shown in Table 4), the best solvent combination for optimal extraction 


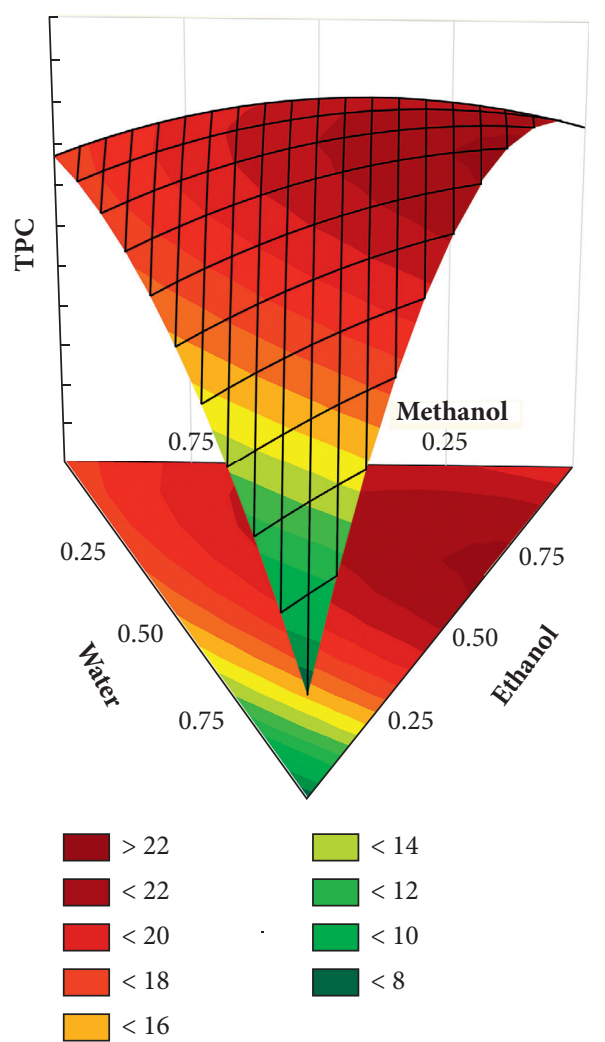

(a)

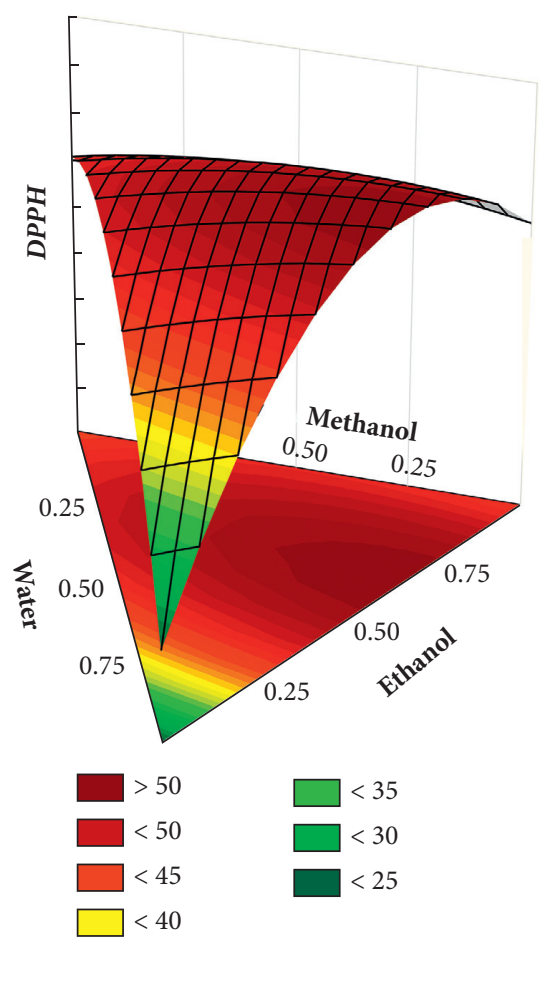

(b)

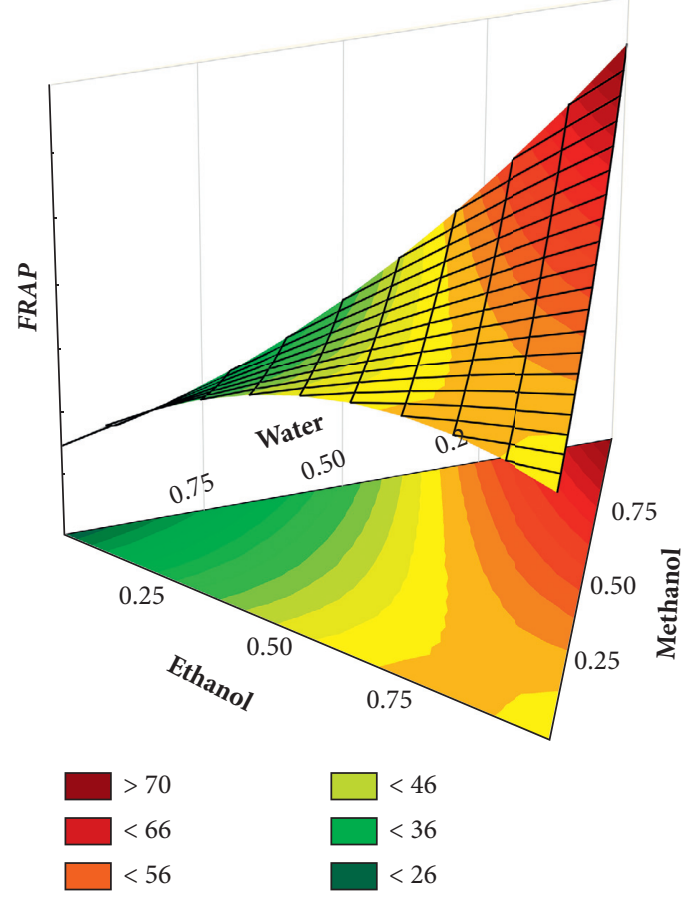

(c)

Figure 2: Mixture contour plots of TPC (a), DPPH (b), and FRAP (c) as a function of proportions of ethanol, methanol, and water.

of the phenolic compound is the ternary mixture which consists of $75 \%$ ethanol, $12.5 \%$ methanol, and $12.5 \%$ water.

Total phenolic compound contour plot, extracted using organic solvent mixtures including ethanol, methanol, and hexane, is shown in Figure 3(a). As shown in Figure 3(a), ethanol extracted the highest amounts of TPC, while hexane was the worst solvent for TPC recovery, which rapidly increases when the proportion of the ethanol increases in the 
TABLE 4: Desirability results.

\begin{tabular}{lcccccc}
\hline & & Hydroalcoholic mixtures & & Organic mixtures \\
& Ethanol & Methanol & Water & Ethanol & Methanol & Hexane \\
\hline TPC & 75 & 12.5 & 12.5 & 50 & 50 & 0 \\
DPPH & 50 & 16.667 & 33.33 & 50 & 50 & 0 \\
FRAP & 0 & 100 & 0 & 0 & 100 & 0 \\
\hline
\end{tabular}

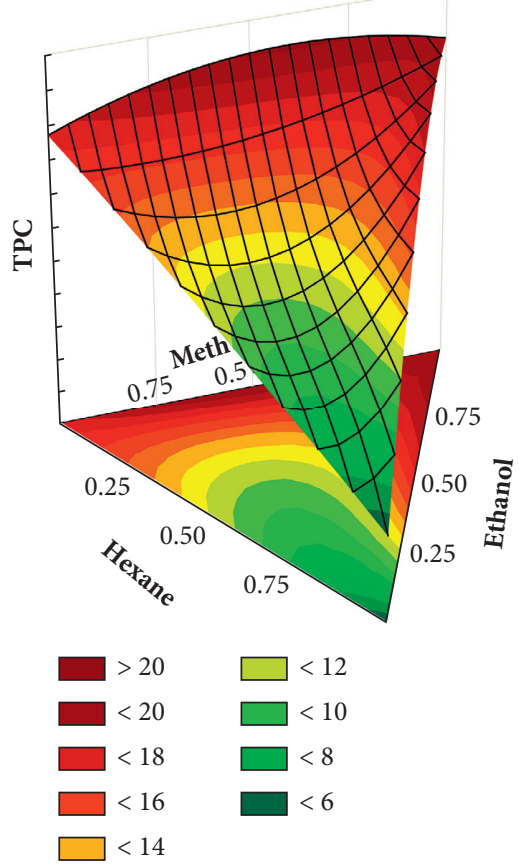

(a)

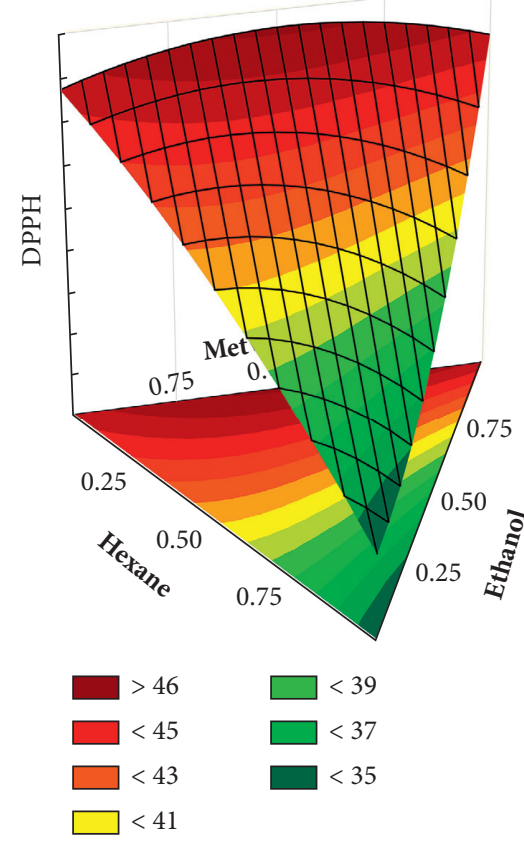

(b)

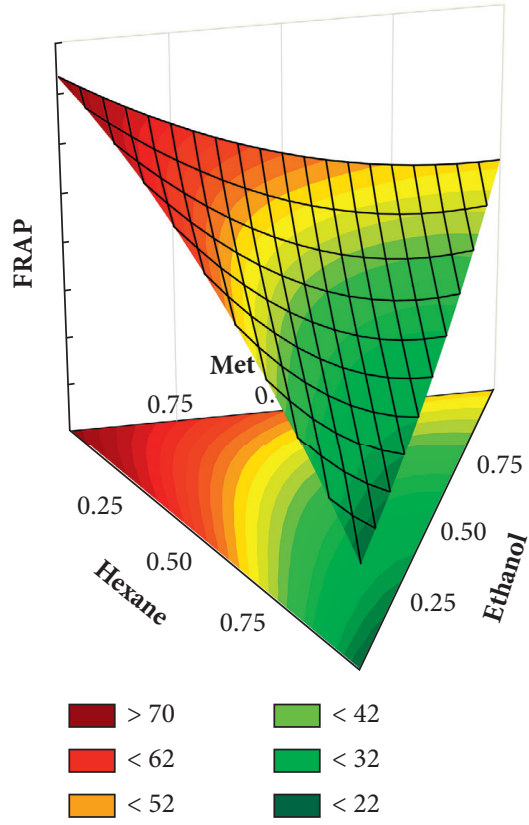

(c)

FIgURE 3: Mixture contour plots of TPC (a), DPPH (b), and FRAP (c) as a function of proportions of ethanol, methanol, and water.

binary mixture. The same observation can be noticed also when adding methanol to hexane, but with less effectiveness. Thus, adding ethanol and methanol to hexane increases its capacity to extract TPC. Desirability analysis (Table 4) revealed the equivalent binary interaction to be the optimal solvent mixture in the absence of water.

3.4.2. DPPH Free Radical Scavenging Activity. Generally, plants bioactive compounds with higher polar character can be easily extracted with water; however highly hydroxylated phenolic compounds, such as catechins, are more soluble in alcohols such as ethanol and methanol [25]. The DPPH assay is employed to test the ability of compounds to act as free radical scavengers and is frequently used to evaluate the antioxidant capacity of foods. The recovery of antioxidants from plant residues is interesting from a technological point of view as valuable components of nutraceuticals in food and pharmaceutical preparations or the cosmetics industry [26].

To investigate the effects of solvents and their interactive effects on antioxidant activity measured by DPPH assay of the extracts, the three-dimensional plots were depicted in Figures 2(b) and 3(b). Concerning the hydroalcoholic mixtures, as evident in Figure 2(b), the DPPH activity values of water extract increased when the methanol or ethanol concentration increases, indicating that adding these organic solvents to water rises its capacity to extract phenolic compounds, although it starts to decrease when ethanol or methanol concentration in the solvent mixture exceeds $75 \%$. The solvent mixtures yielding extracts with the highest DPPH free radical scavenging activity occur between the equivalent ternary mixture, the equivalent binary mixture water-ethanol, and the binary mixture (water 25\%, ethanol $75 \%$ ). Moreover, the optimum solvent mixture given by the desirability analysis (Table 4) was a ternary mixture that consisted of $50 \%$ ethanol, $16.667 \%$ methanol, and $33.33 \%$ water.

Similarly, in the organic mixtures (Figure 3(b)) adding methanol or ethanol to hexane increases its capacity to extract molecules with DPPH free radical scavenging activity. However, in this case the quadratic interaction between methanol and hexane was more effective. The best solvent mixture for yielding extract with optimal DPPH antioxidant activity was the equivalent binary mixture of methanol and ethanol. 


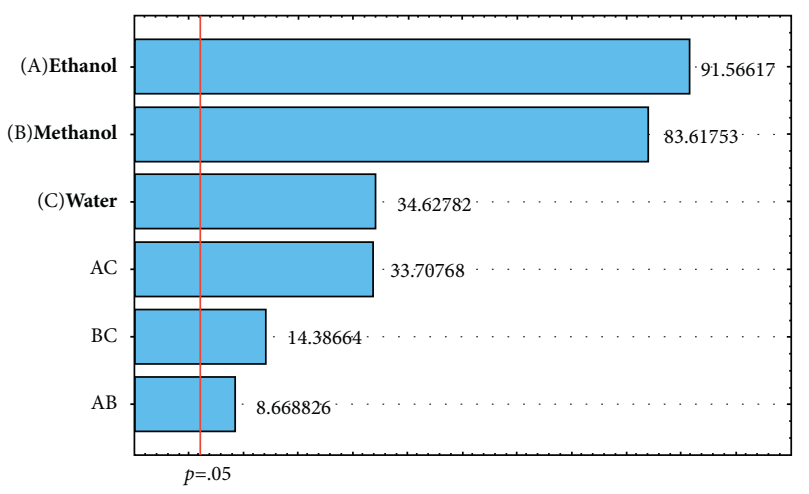

(a)

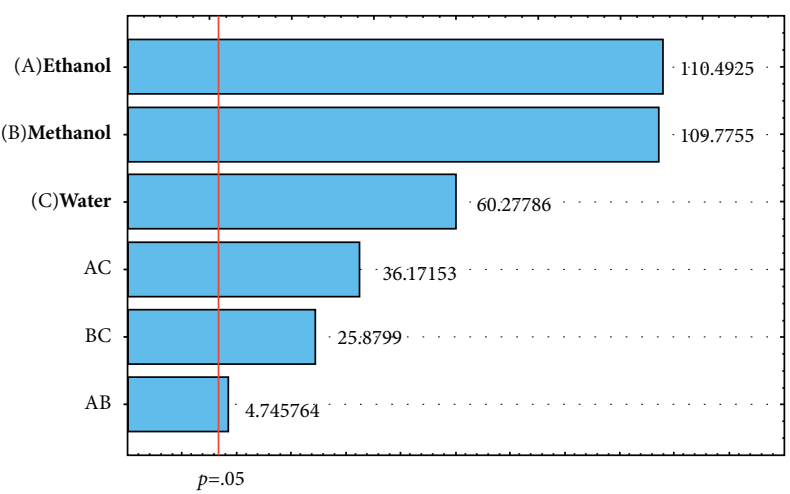

(b)

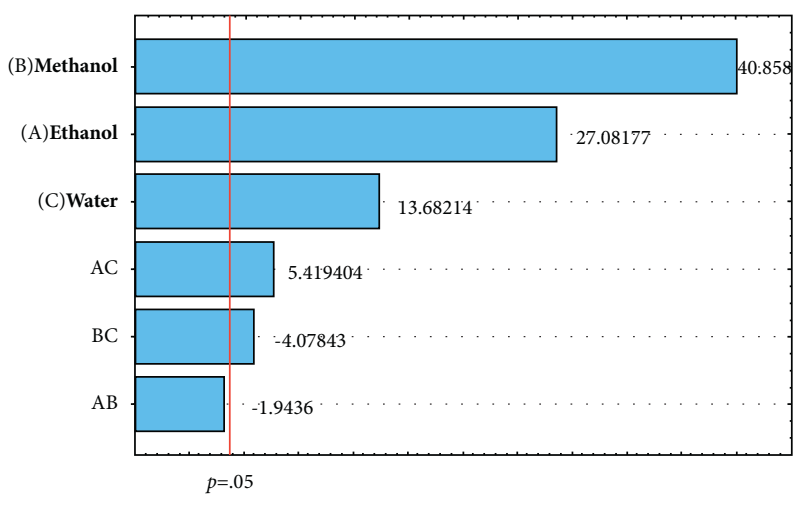

(c)

Figure 4: Analysis of the Pareto chart of the standardized effects for total phenolic content (a), DPPH (b), and FRAP (c) assays.

3.4.3. FRAP Assay. The reductive capacity of the extract may serve as a reflection of its antioxidant activity. The response surface plots of FRAP antioxidant assay are depicted in Figures 2(c) and 3(c), illustrating the combined impact of extraction solvents for hydroalcoholic and organic mixtures, respectively. Unlike methanol, the water and hexane were the less effective solvent. From Figures (2(c) and 3(c)), it can be clearly observed that when the methanol proportion increased in the mixtures of the three solvents (ethanol, methanol, and water), FRAP values increased significantly. The same sightings have been observed when using hexane instead of water. FRAP desirability analysis demonstrated that pure methanol was the optimal solvent for extracting bioactive compounds with FRAP antioxidant activity. Regarding FRAP activity, methanol proportion was one of the most important variables affecting extraction from Thymelaea hirsuta L [27]. According to the planned mixture design performed to extract total anthocyanins, phenolic compounds, and antioxidants from $x$ 'kijit peels, methanol was the solvent producing extracts with the highest extraction yields of total phenolic compounds and other bioactive compounds, exhibiting the highest antioxidant capacity [28].

3.5. Pareto Chart Analysis. To examine the relative importance of the main effects and their interactions using statistical significance $(p<0.05)$, a standardized Pareto chart was employed.
According to Figures 4(a) and 4(b), the result showed the effect of the independent variables (hydroalcoholic mixtures) and their interactions on TPC recovery and DPPH antioxidant activity. We notice for the two responses that all parameter was statistically significant with $95 \%$ confidence and had a positive effect on the two responses and was ranked as follows: ethanol $>$ methanol $>$ water $>$ ethanol ${ }^{*}$ water $>$ methanol ${ }^{*}$ water $>$ ethanol ${ }^{*}$ methanol, whereas in the case of FRAP assay (Figure 4(c)), methanol had the greatest significant positive effect followed by ethanol. The binary interaction methanol ${ }^{*}$ water had a low significant negative effect, meaning that adding water to methanol slightly lowered the recovery on molecules having a FRAP antioxidant activity.

Concerning organic solvent (figures not shown), the Pareto analysis revealed that all parameter was statistically significant with $95 \%$ confidence. In the linear interactions, mostly ethanol exhibited the highest positive influence, followed by methanol; likewise, the quadratic interactions influenced positively the TPC recovery, but with smaller impact, in contrast to ternary interaction which had a negative effect. In the DPPH assay, methanol and ethanol greatly influenced extractions of antioxidant molecules, followed by hexane with lesser effect. The interaction of ethanol-methanol showed a significant negative influence. Methanol was the pure solvent that highly influenced FRAP antioxidant molecules, followed by ethanol. 


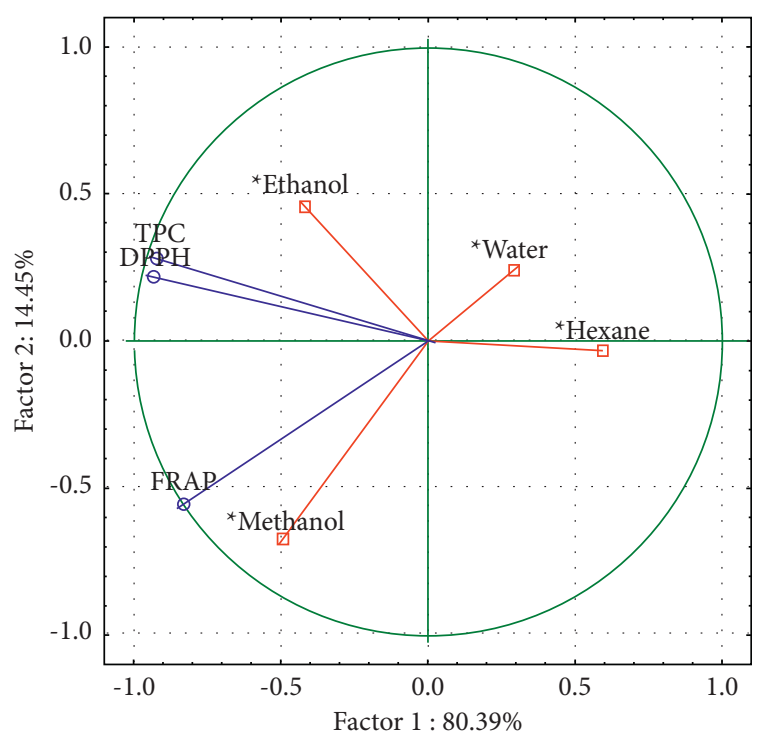

Figure 5: PCA analysis.

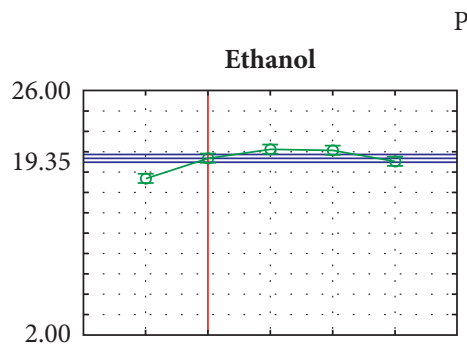

Profiles for Predicted Values and Desirability
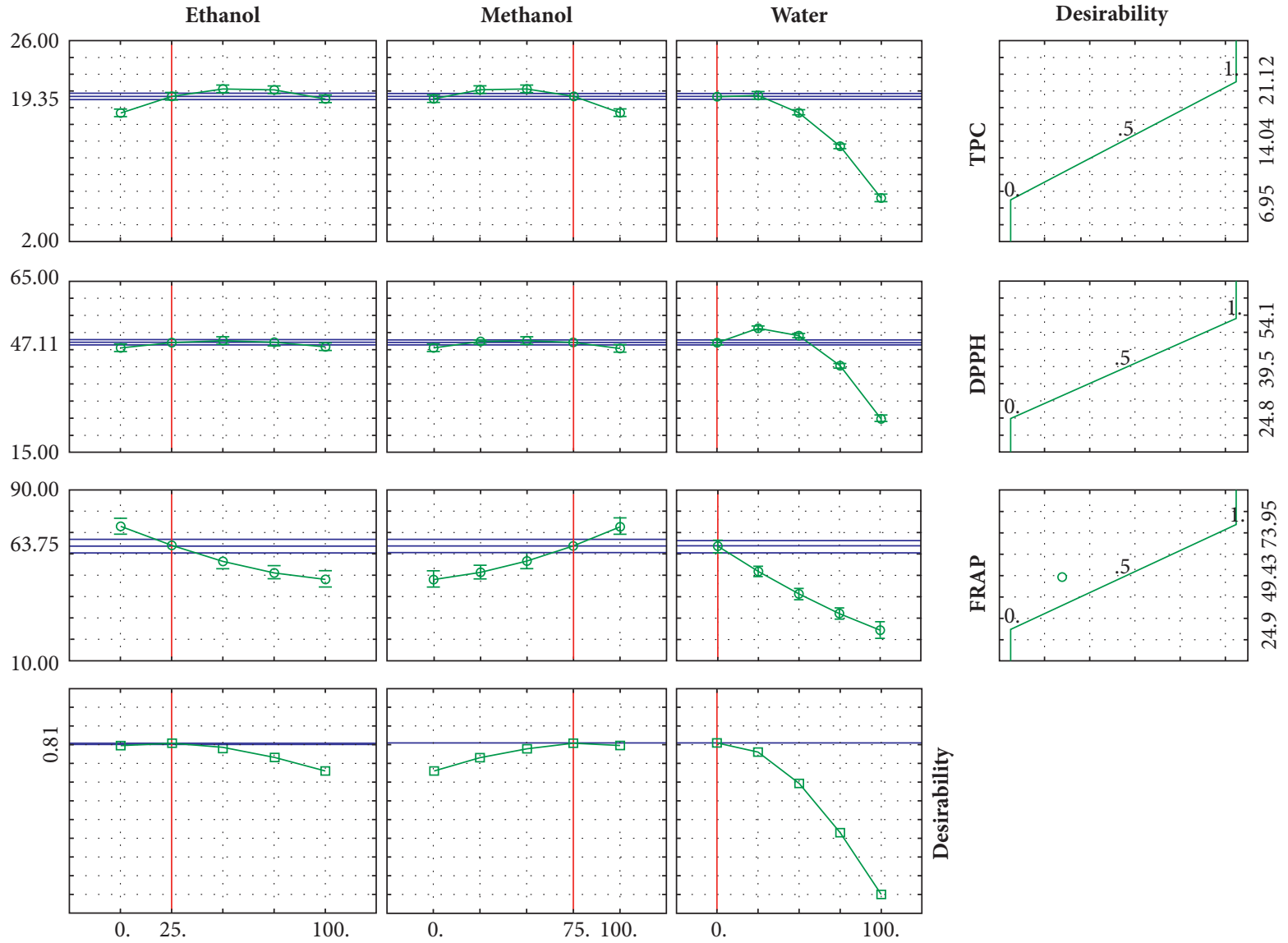

FIgURE 6: Extraction optimization using the desirability function.

3.6. PCA Analysis. Multivariate analysis can summarize the variability of a complex data set and present it in the most interpretable form, such as principal components. PCA results (Figure 5) show that $94.84 \%$ of the variability in the data is accounted by the first two principal components PC1 (Factor 1) and PC2 (Factor 2) which was high enough. 
Furthermore, the first three PCs explained $100 \%$ of the variance in the data, which was high enough to represent all the variables. In this analysis, if two vectors subtend a small angle to each other, this means that the two variables they represent are strongly correlated. The score plot for PC1 versus PC2 clearly shows a strong positive correlation between TPC and DPPH was observed, indicating that fractions with the highest potency in scavenging DPPH radical presented the highest TPC. The two parameters were in turn positively correlated with ethanol concentration in the solvent extractor and negatively correlated to hexane. On the other hand, FRAP shows a positive correlation with methanol concentration in extraction solvent and was negatively correlated with water content, indicating that those ferric reducing molecules have more affinity to methanol than water and ethanol.

3.7. Desirability Analysis. Optimization using the desirability function was performed in order to maximize the total phenolic compounds and also to maximize the antioxidant capacity (DPPH and FRAP) in the extracts. Results are depicted in Figure 6 for hydroalcoholic mixtures. The final result for the simultaneous optimization including all responses, using the desirability function, suggested that the solvent mixture consisting of $25 \%$ ethanol and $75 \%$ methanol was the most adequate solvent to achieve the best solution for this combination of variables. However, pure methanol was the solvent when mixtures were performed with the three organic solvents.

\section{Conclusions}

This is the first report on the optimization of phenolic antioxidants from Cannabis sativa residue, in which we revealed that this plant's waste is a useful source for bioactive compounds. The methods can be utilized for further isolation of active fraction/compounds from the cannabis waste. The highest TPC yield values occur with the binary interaction between water and ethanol around the proportion of ethanol, $70 \%$, water, $30 \%$, whereas the best $\mathrm{DPPH}$ scavenging activity is obtained with extraction using ternary mixtures including water and binary mixture (ethanol 50\% to $75 \%)$. Meanwhile, pure methanol was the best solvent for extracting molecules with FRAP antioxidant capacity.

\section{Data Availability}

The table data used to support the findings of this study are included within the article.

\section{Conflicts of Interest}

The author declares that he has no conflicts of interest.

\section{References}

[1] F. Pellati, V. Brighenti, J. Sperlea, L. Marchetti, D. Bertelli, and S. Benvenuti, "New methods for the comprehensive analysis of bioactive compounds in Cannabis sativa L. (hemp)," Molecules, vol. 23, no. 10, p. 2639, 2018.

[2] V. Cardenia, T. Gallina Toschi, S. Scappini, R. C. Rubino, and M. T. Rodriguez-Estrada, "Development and validation of a fast gas chromatography/mass spectrometry method for the determination of cannabinoids in Cannabis sativa L," Journal of Food and Drug Analysis, vol. 26, no. 4, pp. 1283-1292, 2018.

[3] A. W. Zuardi, "History of cannabis as a medicine: a review," Revista Brasileira de Psiquiatria, vol. 28, no. 2, pp. 153-157, 2006.

[4] P.-A. Chouvy and J. Macfarlane, "Agricultural innovations in Morocco's cannabis industry," International Journal of Drug Policy, vol. 58, pp. 85-91, 2018.

[5] A. Labrousse and L. Romero, Rapport Sur La Situation Du Cannabis Dans Le Rif Marocain, OFDT, Paris, France, 2001.

[6] M. A. El Alaoui, M. Melloul, S. Alaoui Amine et al., "Extraction of high quality DNA from seized Moroccan cannabis resin (hashish)," PLoS One, vol. 8, no. 10, p. e74714, 2013.

[7] D. Ezez and M. Tefera, "Effects of solvents on total phenolic content and antioxidant activity of ginger extracts," Journal of Chemistry, vol. 2021, Article ID 6635199, 5 pages, 2021.

[8] R. Osman and N. Saim, "Selective extraction of organic contaminants from soil using pressurised liquid extraction," Journal of Chemistry, vol. 20138 pages, 2013.

[9] W. Ouedrhiri, M. Balouiri, S. Bouhdid et al., "Mixture design of origanum compactum, origanum majorana and Thymus serpyllum essential oils: optimization of their antibacterial effect," Industrial Crops and Products, vol. 89, pp. 1-9, 2016.

[10] M. C. DiCiaula, G. C. Lopes, I. S. Scarminio, and J. C. P. De Mello, "Optimization of solvent mixtures for extraction from bark of Schinus terebinthifolius by a statistical mixture-design technique and development of a UV-Vis spectrophotometric method for analysis of total polyphenols in the extract," Química Nova, vol. 37, no. 1, pp. 158-163, 2014.

[11] M. Majeed, A. I. Hussain, S. A. S. Chatha et al., "Optimization protocol for the extraction of antioxidant components from origanum vulgare leaves using response surface methodology," Saudi Journal of Biological Sciences, vol. 23, no. 3, pp. 389-396, 2016.

[12] B. Sultana, F. Anwar, and M. Ashraf, "Effect of extraction solvent/technique on the antioxidant activity of selected medicinal plant extracts," Molecules, vol. 14, no. 6, pp. 2167-2180, 2009.

[13] Y. Pan, J. Zhang, T. Shen et al., "Optimization of ultrasonic extraction by response surface methodology combined with ultrafast liquid chromatography-ultraviolet method for determination of four iridoids in Gentiana rigescens," Journal of Food and Drug Analysis, vol. 23, no. 3, pp. 529-537, 2015.

[14] V. L. Singleton, R. Orthofer, and R. M. Lamuela-Raventós, "[14] analysis of total phenols and other oxidation substrates and antioxidants by means of folin-ciocalteu reagent," Methods in Enzymology, vol. 299, pp. 152-178, 1999.

[15] W. Brand-Williams, M. E. Cuvelier, and C. Berset, "Use of a free radical method to evaluate antioxidant activity," $L W T$ Food Science and Technology, vol. 28, no. 1, pp. 25-30, 1995.

[16] M. Oyaizu, "Studies on products of browning reaction. Antioxidative activities of products of browning reaction prepared from glucosamine," The Japanese Journal of $\mathrm{Nu}$ trition and Dietetics, vol. 44, no. 6, pp. 307-315, 1986.

[17] L. M. Z. Garcia, T. F. De Oliveira, P. K. Soares, R. E. Bruns, and I. S. Scarminio, "Statistical mixture design-principal component determination of synergic solvent interactions for 
natural product extractions," Chemometrics and Intelligent Laboratory Systems, vol. 103, no. 1, pp. 1-7, 2010.

[18] C. W. I. Haminiuk, M. S. V. Plata-Oviedo, A. Guedes, A. Stafussa, E. Bona, and S. T. Carpes, "Chemical, antioxidant and antibacterial study of Brazilian fruits," International Journal of Food Science and Technology, vol. 46, no. 7, pp. 1529-1537, 2011.

[19] C. M. Liyana-Pathirana and F. Shahidi, "Antioxidant activity of commercial soft and hard wheat (Triticum aestivum L.) as affected by gastric $\mathrm{pH}$ conditions," Journal of Agricultural and Food Chemistry, vol. 53, no. 7, pp. 2433-2440, 2005.

[20] Z. Drinić, S. Vidović, J. Vladić, A. Koren, B. Kiprovski, and V. Sikora, "Effect of extraction solvent on total polyphenols content and antioxidant activity of Cannabis sativa $L$," Lekovite sirovine, vol. 38, no. 38, pp. 17-21, 2018.

[21] L. Izzo, L. Castaldo, A. Narváez et al., "Analysis of phenolic compounds in commercial cannabis sativa L. inflorescences using UHPLC-Q-orbitrap HRMS," Molecules, vol. 25, no. 3, p. $631,2020$.

[22] Q. D. Do, A. E. Angkawijaya, P. L. Tran-Nguyen et al., "Effect of extraction solvent on total phenol content, total flavonoid content, and antioxidant activity of Limnophila aromatica," Journal of Food and Drug Analysis, vol. 22, no. 3, pp. 296-302, 2014.

[23] I. E. Ksibi, R. B. Slama, K. Faidi, M. B. Ticha, and M. F. M'henni, "Mixture approach for optimizing the recovery of colored phenolics from red pepper (Capsicum annum $L$.) by-products as potential source of natural dye and assessment of its antimicrobial activity," Industrial Crops and Products, vol. 70, pp. 34-40, 2015.

[24] W. H. Wong, W. X. Lee, R. N. Ramanan et al., "Two level half factorial design for the extraction of phenolics, flavonoids and antioxidants recovery from palm kernel by-product," Industrial Crops and Products, vol. 63, pp. 238-248, 2015.

[25] I. C. W. Arts and P. C. H. Hollman, "Optimization of a quantitative method for the determination of catechins in fruits and legumes," Journal of Agricultural and Food Chemistry, vol. 46, no. 12, pp. 5156-5162, 1998.

[26] A. C. Santos Felix, C. G. Novaes, M. Pires Rocha, G. E. Barreto, B. B. Do Nascimento, and L. D. Giraldez Alvarez, "Mixture design and Doehlert matrix for the optimization of the extraction of phenolic compounds from spondias mombin L apple bagasse agroindustrial residues," Frontiers in Chemistry, vol. 5, 2018.

[27] M. Yahyaoui, N. Ghazouani, S. Saoudi, I. Sifaoui, N. Chammem, and M. Abderrabba, "Experimental design methodology application in the optimization of phytochemical compounds extraction and antioxidant activity of Thymelaea hirsuta L. extracts," Journal of Materials and Environmental Science, vol. 9, no. 5, pp. 1551-1561, 2018.

[28] A. Javier David Vega, R.-E. Hector, L.-G. Juan Jose et al., "Effect of solvents and extraction methods on total anthocyanins, phenolic compounds and antioxidant capacity of Renealmia alpinia (Rottb.) Maas peel," Czech Journal of Food Sciences, vol. 35, no. 5, pp. 456-465, 2017. 